\title{
Single-cell RNA-seq of human induced pluripotent stem cells reveals cellular heterogeneity and cell state transitions between subpopulations
}

\author{
Quan H. Nguyen, ${ }^{1,4}$ Samuel W. Lukowski, ${ }^{1,4}$ Han Sheng Chiu, ${ }^{1}$ Anne Senabouth, ${ }^{1}$ \\ Timothy J.C. Bruxner, ${ }^{1}$ Angelika N. Christ, ${ }^{1}$ Nathan J. Palpant, ${ }^{1,4}$ \\ and Joseph E. Powell $1,2,3,4$
}

${ }^{1}$ Institute for Molecular Bioscience, University of Queensland, Brisbane, Queensland, 4072, Australia; ${ }^{2}$ Garvan-Weizmann Centre for Cellular Genomics, Garvan Institute of Medical Research, New South Wales, 2010, Australia; ${ }^{3}$ St Vincent's Clinical School, UNSW Sydney, New South Wales, 2010, Australia

\begin{abstract}
Heterogeneity of cell states represented in pluripotent cultures has not been described at the transcriptional level. Since gene expression is highly heterogeneous between cells, single-cell RNA sequencing can be used to identify how individual pluripotent cells function. Here, we present results from the analysis of single-cell RNA sequencing data from 18,787 individual WTC-CRISPRi human induced pluripotent stem cells. We developed an unsupervised clustering method and, through this, identified four subpopulations distinguishable on the basis of their pluripotent state, including a core pluripotent population (48.3\%), proliferative (47.8\%), early primed for differentiation $(2.8 \%)$, and late primed for differentiation (1.1\%). For each subpopulation, we were able to identify the genes and pathways that define differences in pluripotent cell states. Our method identified four transcriptionally distinct predictor gene sets composed of 165 unique genes that denote the specific pluripotency states; using these sets, we developed a multigenic machine learning prediction method to accurately classify single cells into each of the subpopulations. Compared against a set of established pluripotency markers, our method increases prediction accuracy by $10 \%$, specificity by $20 \%$, and explains a substantially larger proportion of deviance (up to threefold) from the prediction model. Finally, we developed an innovative method to predict cells transitioning between subpopulations and support our conclusions with results from two orthogonal pseudotime trajectory methods.
\end{abstract}

[Supplemental material is available for this article.]

The transcriptome is a key determinant of the phenotype of a cell and regulates the identity and fate of individual cells. Much of what we know about the structure and function of the transcriptome comes from studies averaging measurements over large populations of cells, many of which are functionally heterogeneous. Such studies conceal the variability between cells and so prevent us from determining the nature of heterogeneity at the molecular level as a basis for understanding biological complexity. Cell-tocell differences in any tissue or cell culture are a critical feature of their biological state and function.

In recent decades, the isolation of pluripotent stem cells, first in mouse followed by human (Evans and Kaufman 1981; Thomson et al. 1998), and the more recent discovery of deriving pluripotent stem cells from somatic cell types (iPSCs) (Takahashi and Yamanaka 2006), is a means to study lineage-specific mechanisms underlying development and disease to broaden our capacity for biological therapeutics (Palpant et al. 2017). Pluripotent stem cells are capable of unlimited self-renewal and can give rise to specialized cell types based on stepwise changes in the transcriptional networks that orchestrate complex fate choices from pluripotency into differentiated states.

\footnotetext{
${ }^{4}$ These authors contributed equally to this work. Corresponding author: j.powell@garvan.org.au Article published online before print. Article, supplemental material, and publication date are at http://www.genome.org/cgi/doi/10.1101/gr.223925.117.
}

In addition to individual published data, international consortia are banking human induced pluripotent stem cells (hiPSCs) and human embryonic stem cells (hESCs) and providing extensive phenotypic characterization of cell lines including transcriptional profiling, genome sequencing, and epigenetic analysis as data resources (The Steering Committee of the International Stem Cell Initiative 2005; Streeter et al. 2017). These data provide a valuable reference point for functional genomics studies but continue to lack key insights into the heterogeneity of cell states that represent pluripotency.

Although transcriptional profiling has been a common endpoint for analyzing pluripotency, the heterogeneity of cell states represented in pluripotent cultures has not been described at a global transcriptional level. Since each cell has a unique expression state comprising a collection of regulatory factors and target gene behavior, single-cell RNA sequencing (scRNA-seq) can provide a transcriptome-level understanding of how individual cells function in pluripotency (Wen and Tang 2016). These data can also reveal insights into the intrinsic transcriptional heterogeneity comprising the pluripotent state. In this study, we provide the largest data set of single-cell transcriptional profiling of undifferentiated hiPSCs currently available, which cumulatively amount to

(C) 2018 Nguyen et al. This article is distributed exclusively by Cold Spring Harbor Laboratory Press for the first six months after the full-issue publication date (see http://genome.cshlp.org/site/misc/terms.xhtml). After six months, it is available under a Creative Commons License (Attribution-NonCommercial 4.0 International), as described at http://creativecommons.org/licenses/bync/4.0/. 
18,787 cells across five biological replicates. Moreover, we developed several innovative single-cell methods focused on unbiased clustering, machine learning classification, and quantitative and directional cellular trajectory analysis. Our findings address the following hypotheses: (1) Pluripotent cells form distinct groups or subpopulations of cells based on biological processes or differentiation potential; (2) transcriptional data at single-cell resolution reveal gene networks governing specific cell subpopulations; and (3) transcripts can exhibit differences in gene expression heterogeneity between specific subpopulation of cells.

\section{Results}

\section{Description of the parental hiPSC line, CRISPRi}

WTC-CRISPRi hiPSCs (Mandegar et al. 2016) were chosen as the parental cell line for this study. These cells are genetically engineered with an inducible nuclease-dead Cas9 fused to a KRAB repression domain (Supplemental Fig. S1A). Transcriptional inhibition by gRNAs targeted to the transcriptional start site is doxycycline-dependent and can be designed to silence genes in an allelespecific manner. The versatility of this line provides a means to use this scRNA-seq data as a parental reference point for future studies aiming to assess the transcriptional basis of pluripotency at the single-cell level. Cells were verified to have a normal $46 \mathrm{X}, \mathrm{Y}$ male karyotype by Giemsa banding analysis before analysis by scRNA-seq (Supplemental Fig. S1B).

\section{Overview of single-cell RNA sequence data}

After quality control of the sequencing data (Methods), we obtained 1,030,909,022 sequence reads for 20,482 cells from five hiPSC single-cell samples (Supplemental Fig. S2; Supplemental Table S1), with 63\%-71\% confidently and uniquely mapped (mapping quality 255) to the human reference transcriptome hg19 (Ensembl, release 75). We sequenced 19,937 cells from four samples to an average depth of 44,506 reads per cell (RPC), and one sample consisting of 545 cells was sequenced to an average depth of 318,909 RPC. On average, 2536 genes and 9030 unique molecular identifiers (UMIs) were detected per cell. Comparing the sequencing results among five samples, we observe little variation in the total number of genes detected per sample, despite differences in the number of cells per sample, and average reads per cell (Supplemental Table S1). For example, we observed only a slight increase in the average number of genes detected for cells sequenced at a greater depth (Supplemental Fig. S2F; Supplemental Tables S1, S3) and no gain in the total number of genes detected for all cells in the whole sample. These results suggest that an average of 44,506 RPC achieves close to maximum total gene detection in our samples. We found that the number of reads per cell affects per-cell gene detection (sensitivity), whereas the number of cells per sample effects the total gene detection (more unique genes per sample). Overall, after quality control, we detected 16,064 unique genes, which were expressed in at least $1 \%$ of the total cells. Importantly, of the 16,064 genes, only one was unique to a single sample (Supplemental Table S4). We subsequently removed 1738 cells due to a high percentage of expressed mitochondrial and/or ribosomal genes (Supplemental Table S2; Methods), leaving a total of 18,787 high-quality hiPSCs for further analysis. Following between-sample and between-cell normalization, we observed no evidence for batch effects due to sample or sequencing run (Fig. 1A; Supplemental Fig. S3).

\section{Identification of four hiPSC subpopulations based on biological} function

We developed an innovative classification method, which we term unsupervised high-resolution clustering (UHRC), to objectively assign cells into subpopulations based on genome-wide transcript levels (Fig. 1; Supplemental Fig. S4; Methods). The UHRC procedure comprises three unbiased algorithms. First, a PCA reduction step was implemented to overcome the inherent multicollinearity in single-cell expression data. Subsequently, we applied bottom-up agglomerative hierarchical clustering, which importantly provides a "data-driven" identification of clusters, rather than inputting a specific number of expected clusters, as is the case with $K$-means algorithms. Third, to robustly define large clusters, a dynamic branch merging process was used to detect complex nested structures and outliers. This unbiased method identified four independent subpopulations of cells containing $48.3 \%, 47.8 \%, 2.8 \%$, and $1.1 \%$ of the 18,787 cells, respectively (Fig. 1A,B,D; Supplemental Fig. S4). Importantly, after clustering, we did not observe evidence for batch effects underlying any of the four cell subpopulations (Fig. 1A; Supplemental Table S5), suggesting that the clusters represent biological and not technical factors. By comparing gene expression levels between subpopulations, we identified four differentially expressed gene sets that distinguish each subpopulation from the remaining cells (Fig. 1C; Supplemental Table S6). We have made this data available via an interactive, gene-searchable web application available at http://computationalgenomics.com.au/shiny/hipsc/.

We initially examined transcript dynamics in the subpopulations based on expression of previously described markers of pluripotency and lineage determination (Fig. 2; Supplemental Table S7; Tsankov et al. 2015). Of the 18,787 cells, $99.8 \%$ expressed at least one of 19 pluripotency genes (Supplemental Table S8). Furthermore, genes with known roles in pluripotency had stronger expression across all subpopulations compared to genes involved in lineage determination (Fig. 2A,B; Supplemental Tables S7, S8). For example, POU5F1 (also known as OCT4), which encodes a transcription factor critically involved in the self-renewal of undifferentiated pluripotent stem cells (Boyer et al. 2005), was consistently expressed in $98.6 \%$ of cells comprising all four subpopulations (Fig. 2A,B; Supplemental Tables S7, S8). Other known markers of pluripotency such as SOX2, NANOG, and UTF1 were expressed across the subpopulations (Fig. 2A,B; Supplemental Tables S7, S8), but showed differences in expression heterogeneity, suggesting differences in the pluripotent state across subpopulations (Supplemental Table S7).

\section{Classification of hiPSC subpopulations}

We sought to identify biological processes underlying transcriptional classification of cell subpopulations by first performing a statistical analysis to identify significantly differentially expressed genes between subpopulations (Fig. 1C; Supplemental Table S6; Methods). Differentially expressed genes with a fold-change significant at a Bonferroni-corrected $P$-value threshold $\left(P<3.1 \times 10^{-7}\right)$ were evaluated for enrichment of functional pathways (Supplemental Tables S9-S13).

Cells classified in subpopulations one and two, which make up $96.1 \%$ of total cells analyzed (Fig. 1A-C), were distinguished from one another by significantly different expression levels of genes in alternate pathways controlling pluripotency and differentiation (Supplemental Fig. S5; Supplemental Tables S9S11). The Transcriptional Regulation of Pluripotent Stem Cells (TRPSC) pathway was consistently up-regulated in cells classified

\section{Genome Research}

www.genome.org 
A

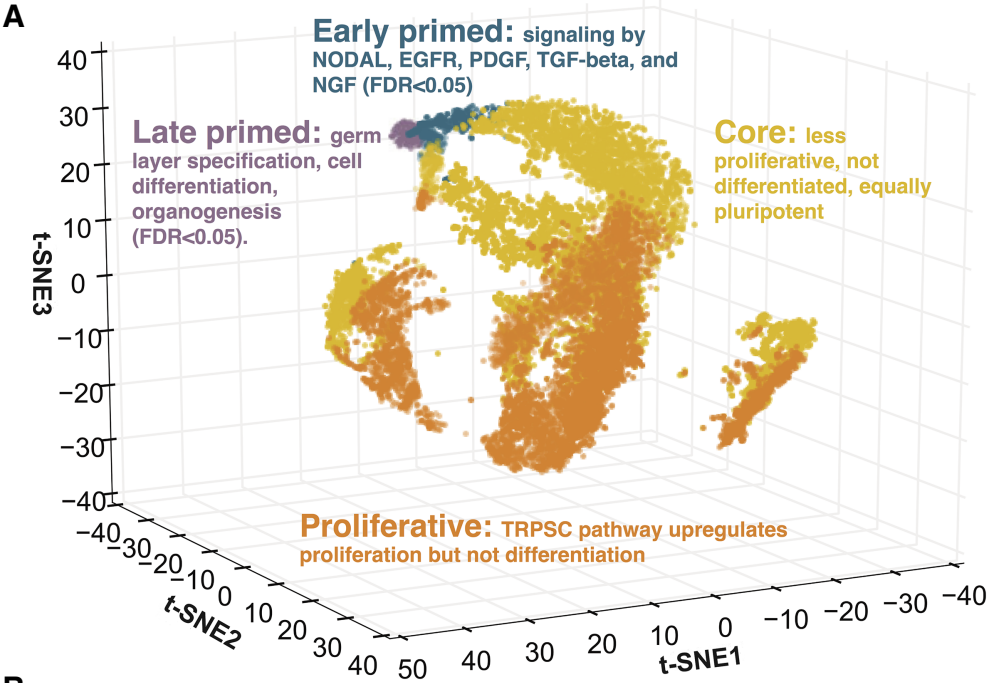

B

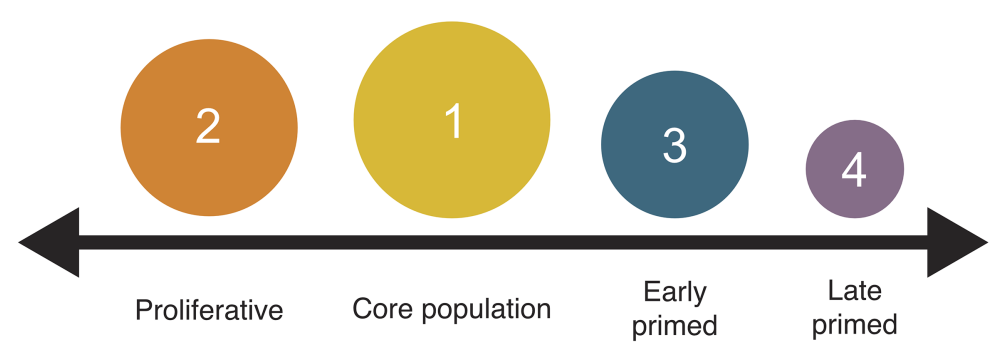

Subpop 1 vs Subpops 2, 3, 4
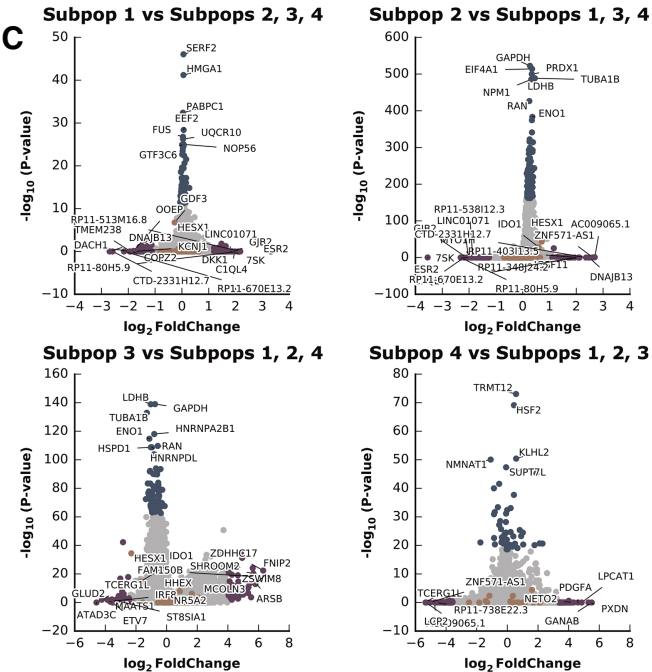

D

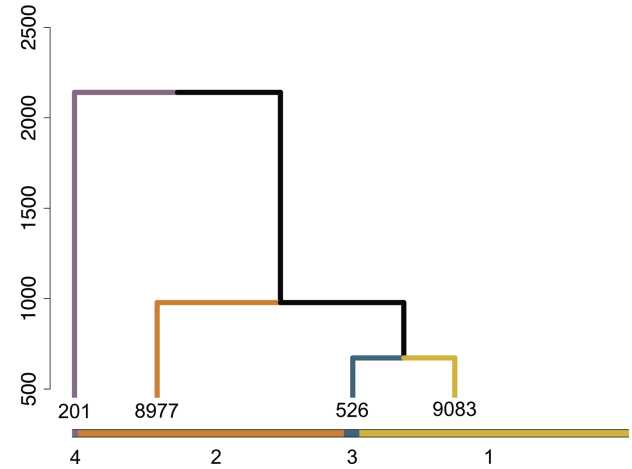

Figure 1. Identification of four cell subpopulations from 18,787 hiPSC cells, sequenced from five biological replicates. ( $A$ ) Three-dimensional t-SNE distribution of cells based on gene expression value. Each point represents a single cell in three-dimensional space. A t-SNE transformation of the data was used for positioning cells; four cell subpopulation labels (marked by different colors) represent results from clustering and are independent of t-SNE data transformation (for an interactive, searchable figure, see http://computationalgenomics.com.au/shiny/hipsc/). Pathway analysis based on differential expression identified functional properties that distinguish each subpopulation. (B) Four pluripotent subpopulations functionally separated from a homogeneous hiPSC population. (C) The top significantly differentially expressed genes of cells in a subpopulation compared to cells in the remaining three subpopulations. Genes denoted with orange points are known naive and primed markers. Genes represented with blue and purple points are those in the top $0.5 \%$ highest $\log F C$ or $-\log (P$-value), respectively. $(D)$ Unsupervised clustering of all cells into four subpopulations. The dendrogram tree displays distance and agglomerative clustering of the cells. Each branch represents one subpopulation. The clustering is based on a Dynamic Tree Cut that performs a bottom-up merging of similar branches. The number of cells in each of the four subpopulations are given below the branches.

as subpopulation two compared to subpopulation one (Supplemental Fig. S5; Supplemental Tables S11, S14). TRPSC is an auto-activation loop, which maintains expression of POU5F1, NANOG, and SOX2 at high levels. Complexes containing various combinations of these transcription factors (Lam et al. 2012) can activate the expression of genes whose products are associated with rapid cell proliferation, and they also repress the expression of genes associated with cell differentiation (Supplemental Fig. S5; Forristal et al. 2010; Guenther 2011). In particular, POU5F1, NANOG, and SOX2 are more highly expressed in subpopulation two (Supplemental Table S7), and the direction of differential expression of genes associated with cell proliferation and repression of cell differentiation (Forristal et al. 2010; Guenther 2011) is consistent with subpopulation two containing cells that are more active in their self-renewal than cells in subpopulation one (Supplemental Tables S7, S10, S14).

We hypothesized that expression differences between subpopulations one and two could reveal novel, subpopulationspecific markers. We applied unbiased differential expression analysis between subpopulation one and two, identifying 49 stat- istically significant genes that were taken forward to biological pathway analysis (Supplemental Fig. S5; Supplemental Table S6). Among these 49 genes, two key transcription factors and a signaling receptor were significantly higher in subpopulation two than subpopulation one, including SALL4 (spalt-like transcription factor 4, $P$-adjusted: $7.0 \times 10^{-5}$ ), ZIC1 (Zic family member $1, P$-adjusted: $4.3 \times 10^{-5}$ ), and NR6A1 (nuclear receptor subfamily 6 group A member 1, $P$-adjusted: $3.7 \times 10^{-6}$ ) (Supplemental Table S14).

SALL4 is one of the key transcription factors that participates in controlling transcriptional balance in pluripotent cells and suppressing differentiation (Supplemental Fig. S5; Miller et al. 2016). Specifically, SALL4 activates transcription of POU5F1 and maintains pluripotency (Yang et al. 2010b). ZIC1, an up-regulated gene in subpopulation two, was identified by GeneMANIA (Warde-Farley et al. 2010) analysis to be related to SALL4 through shared protein domains (Supplemental Fig. S6). Both ZIC1 and SALL4 were predicted by the STRING database (Szklarczyk et al. 2015) to interact with key pluripotency markers (Supplemental Fig. S6). Furthermore, ZIC1 and its paralog ZIC3, a key member in the TRPSC pathway (Supplemental Fig. S5), are involved in 
A
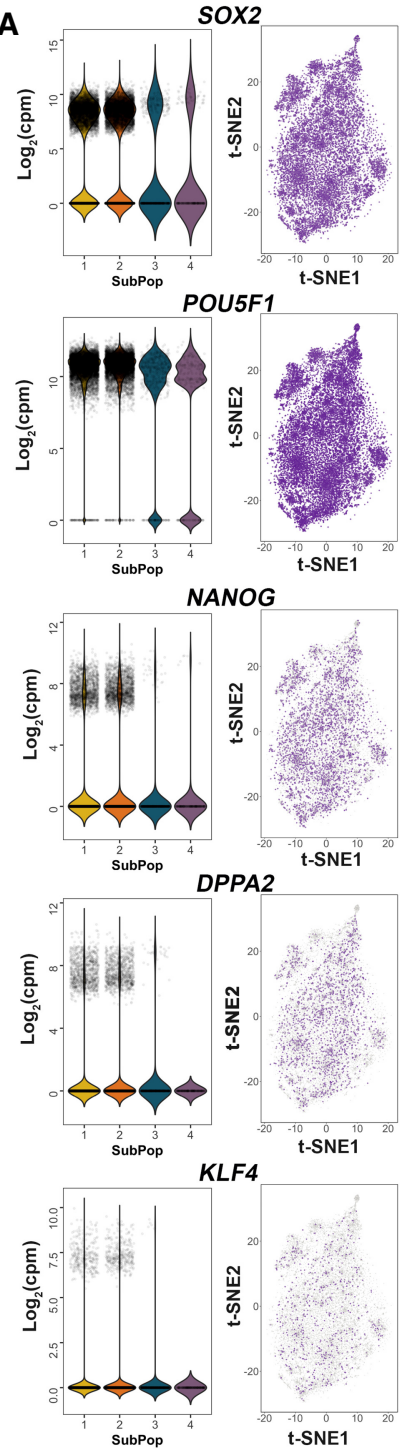
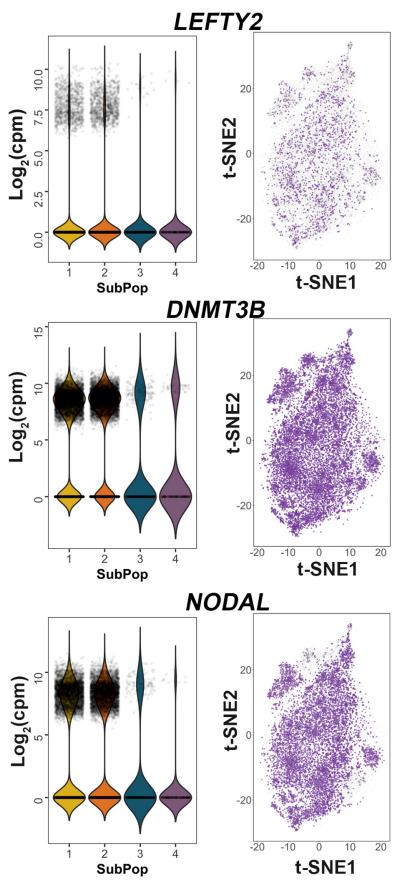

B
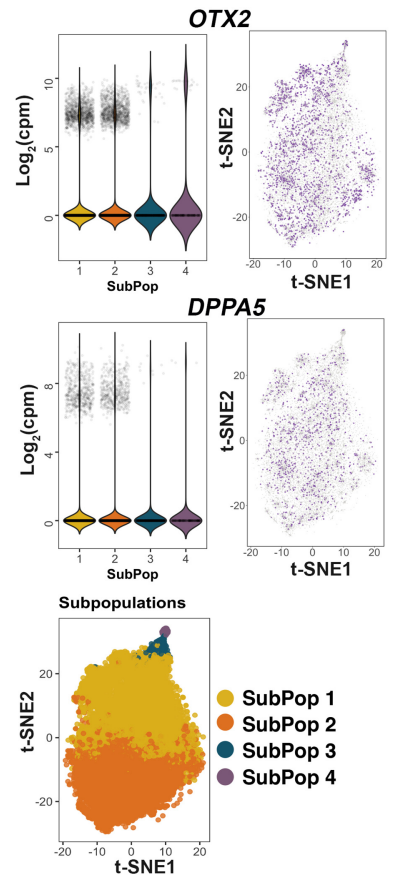

Known Pluripotency Markers
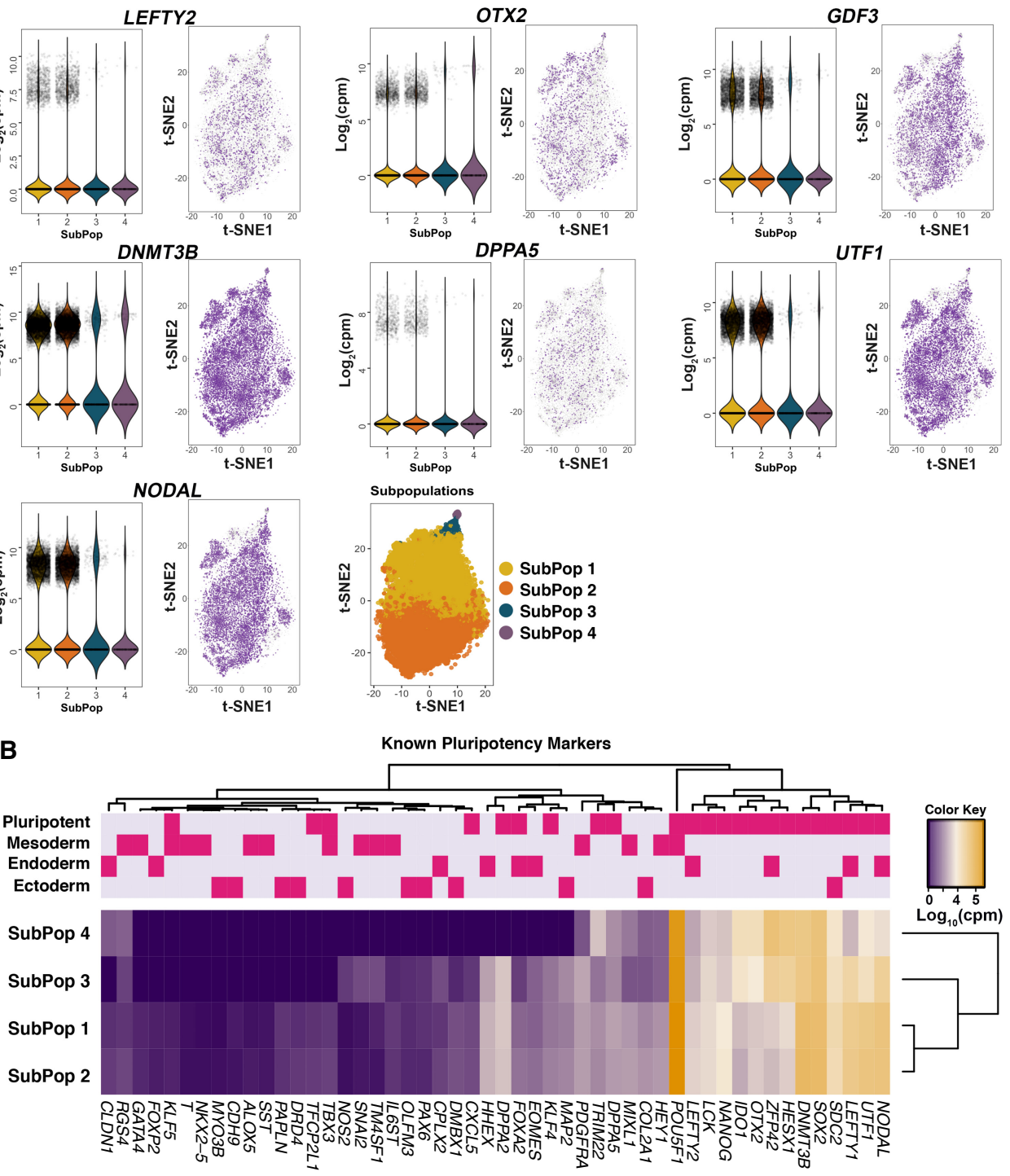

Figure 2. Expression levels of known pluripotency and lineage-primed markers. $(A)$ Violin and jitter plots and t-SNE plots for expression of top pluripotency markers. Each point represents a single cell. The color gradient in the t-SNE plot represents the relative expression level of the gene in a cell across the whole population and subpopulations: (light gray) low; (dark purple) high. (B) Heatmap of the mean expression of known markers within each subpopulation. The upper panel shows the classifications of genes into pluripotency and lineage-primed markers.

maintaining the undifferentiated state, for example in the case of neural precursor cells (Inoue et al. 2007). Moreover, we also identified another differentially expressed gene, NR6A1, a known regulator of POU5F1 later in differentiation (Fuhrmann et al. 2001; Weikum et al. 2016), and which we predict is likely to participate in the TRPSC pathway since its paralog, NR5A1, is among the key members of this pathway (Supplemental Fig. S6). Based on these observations, we hypothesize that in subpopulation two, three differentially expressed (DE) genes-SALL4, NR6A1, and ZIC3-cooperate with key pluripotency transcription factors POU5F1, SOX2, and NANOG to activate genes related to proliferation, but not genes involved in differentiation (Supplemental Fig. S5).

Compared to subpopulations one and two, subpopulations three and four represent pluripotent populations with significant down-regulation of key pluripotency network genes (e.g., NANOG and UTF1) (Fig. 2A,B). For all known pluripotency markers examined in Supplemental Table S8, we observed expression in a higher proportion of cells in subpopulation one and two than subpopulations three and four. Furthermore, the mean expression of 46 of 56 known markers involved in pluripotency shown in Supplemental Table S7 is higher in subpopulations one and two than in three and four. For subpopulation three, comprising $2.8 \%$ of cells, Reactome pathway enrichment analysis of 2534 DE genes between subpopulations three and four showed the top pathways related to developmental signaling and transcriptional regulation via chromatin modification (Supplemental Table S12). Intracellular signaling pathways that control cell proliferation, cell differentiation, and cell migration, such as EGFR, PDGF, and NGF pathways $\left(\right.$ FDR $\left.<1.7 \times 10^{-6}\right)$, were the top three most enriched pathways (Supplemental Table S12). Additionally, signaling pathways by FGFRs involved in differentiation were also significantly enriched (FDR $\left.<3 \times 10^{-4}\right)$. Comparing subpopulations three and one, signaling 
by TGF-beta, and signaling by NODAL were in the top enriched pathways (FDR $<8 \times 10^{-3}$ ). Similarly, signaling by NODAL $(\mathrm{FDR}<$ 0.04) (LeVincent et al. 2003) and pre-NOTCH processing (FDR < 0.04) (Artavanis-Tsakonas et al. 1999), which are involved in cell fate decisions, were in the top enriched pathways when comparing subpopulation three to subpopulations one and two (Supplemental Table S12). In addition, "chromatin modifying enzymes" is among the top Reactome pathways distinguishing subpopulation three, suggesting that cells are undergoing active transcription regulation. Thus, pluripotent cells in subpopulation three appear more lineage primed compared to subpopulations one and two.

Further, we extended the pathway enrichment analysis to a broad collection of background Gene Ontology databases, beyond the manually curated Reactome database (Wu et al. 2010), by using BiNGO in Cytoscape (Maere et al. 2005) for all 1706 DE genes in subpopulation four versus all other subpopulations (1.1\% of analyzed cells) (Supplemental Table S13). We found the top enriched pathways related to differentiation including genes involved in gastrulation $\left(\mathrm{FDR}<1.3 \times 10^{-2}\right)$ and formation of primary germ layer $\left(\mathrm{FDR}<1.4 \times 10^{-2}\right)$, developmental process $\left(\mathrm{FDR}<2.8 \times 10^{-3}\right.$ ) and cell differentiation (FDR $<1.2 \times 10^{-2}$ ), and more than 20 significantly enriched pathways related to organogenesis (FDR $<5 \times$ $10^{-2}$ ) (Supplemental Table S13). Thus, although cells in subpopulation four are still pluripotent, as indicated by the expression of pluripotent markers, they likely represent cells at a late primed state progressing toward differentiation.

Taken together, our transcriptional profiling of single cells revealed four subpopulations defined by their pluripotency levels, cell proliferation, and potential for cell lineage commitment. Subpopulation one pluripotent cells likely represent a core pluripotent state, subpopulation two proliferative pluripotent cells, subpopulation three as early primed for differentiation, and subpopulation four as late primed for differentiation (Fig. 1B).

\section{Improved cell classification using a reduced set of gene markers}

Current methods of classifying cells into subtypes or functional groups rely on prior knowledge of so-called "marker genes" to inform the pseudotime algorithm. One limitation of this approach is that to discover new or rare cell types using single-cell RNAseq, the use of established markers as an input may not be feasible.

Using differentially expressed genes identified between subpopulations, we built a novel unbiased machine learning predictor to identify the pluripotency potential of a single cell. To avoid overfitting the model due to coexpression of genes, we used a variable selection regression model called LASSO (Tibshirani 1996) to estimate gene effects differentiating each subpopulation conditional upon the effects of other genes. Using a 100-fold bootstrapping approach, we estimated the predictive accuracy of identifying a cell in each of the four subpopulations (Fig. 3; Supplemental Table S15; Tibshirani 1996). To detect new gene markers compared to the use of known pluripotency markers (Supplemental Table S7), we applied LASSO to selected sets of differentially expressed genes between one subpopulation compared to the remaining subpopulations. Consistently across four comparisons, our method increases prediction accuracy by $10 \%$, specificity by $20 \%$, and explains a substantially larger proportion of deviance (up to threefold) from the prediction model compared to known markers (Supplemental Figs. S7, S8; Supplemental Tables S15, S16). Prediction by DE genes showed markedly higher performance, measured specifically by sensitivity, specificity, and percentage of deviance explained, in distinguishing cells classified in sub- populations three and four from the remaining cells (Fig. 3A). Similarly, a significantly higher percentage of deviance can be explained by using DE genes compared to using known markers ( $t$-test, $P=1.4 \times 10^{-77}$ ) (Fig. 3B). We observed the highest classification accuracy using genes identified using the LASSO model for cells in subpopulations three and four than cells in subpopulations one and two, suggesting that these subpopulations were more divergent from the remaining majority of the cell population (Fig. 3C). This observation further supports the classification of subpopulations three and four as more primed to differentiation than subpopulations one and two. Although the difference in accuracy was less apparent for cells in subpopulations one and two, the improved accuracy achieved using the DE genes compared to known markers supports the conclusion that our analysis has identified novel genes that act as pluripotency markers (Fig. 3C).

\section{Cell transition between pluripotency states}

We next sought to investigate cell trajectories between subpopulations of iPSCs. We further deployed the results from the LASSO variable selection procedure described above to develop a classifier model, termed Local Transitions between Subpopulations (LTS) that can predict the conversion potential of cells in one subpopulation into cells in a target subpopulation. For each subpopulation, we trained the expression data using the genes identified as differentially expressed to optimize a logistic regression model by penalized maximum likelihood estimation. The training provides an optimal LASSO model with selected genes and corresponding coefficients. The coefficients estimated from the training model were fitted into the expression levels of each of the remaining subpopulations (target populations), enabling us to predict which cells in the target population were the most similar to cells in the training subpopulation. The percentage of cells within the target subpopulation accurately predicted by the training subpopulation is termed the transition score. The transition scores provide both a quantitative and directional estimate of the progression potential between subpopulations. Using LTS to predict the lineage trajectory between subpopulations of pluripotent cells showed that subpopulation one is capable of progressing into all other states, with the potential to convert into $45 \%, 69 \%$, and $47 \%$ of the cells in the subpopulation two, three, and four, respectively (Fig. 4E; Table 1). In contrast, subpopulation two predominantly transitions into subpopulation one (39\%), with low transition scores when compared against subpopulations three and four $(<5 \%)$ (Fig. $4 \mathrm{E})$. The difference in transition scores from subpopulation two to other subpopulations suggests that the cells were at a reversible, selfrenewal state (Table 1). Notably, the LASSO method predicts the highest transition score for the progression from subpopulation three to subpopulation four ( $>99 \%$ ), providing further support that subpopulation three is "early primed" prior to the "late primed" state in subpopulation four (Fig. 4E).

To confirm that the population of iPSCs consists of groups of cells located in different states along a differentiation path, we cross-validated our findings with two independent pseudotime analysis methods, namely Monocle 2 (Qiu et al. 2017) and diffusion pseudotime (Haghverdi et al. 2016). These two methods estimate the differentiation distance of a cell compared to a root cell (Methods), but do not otherwise provide quantitative information about direction of movement. Monocle 2 (Fig. 4A,B) revealed a substantial overlap between subpopulations one and two, with cells classified in subpopulation one distributed across the trajectory, indicating a pluripotent state. Furthermore, the majority of 

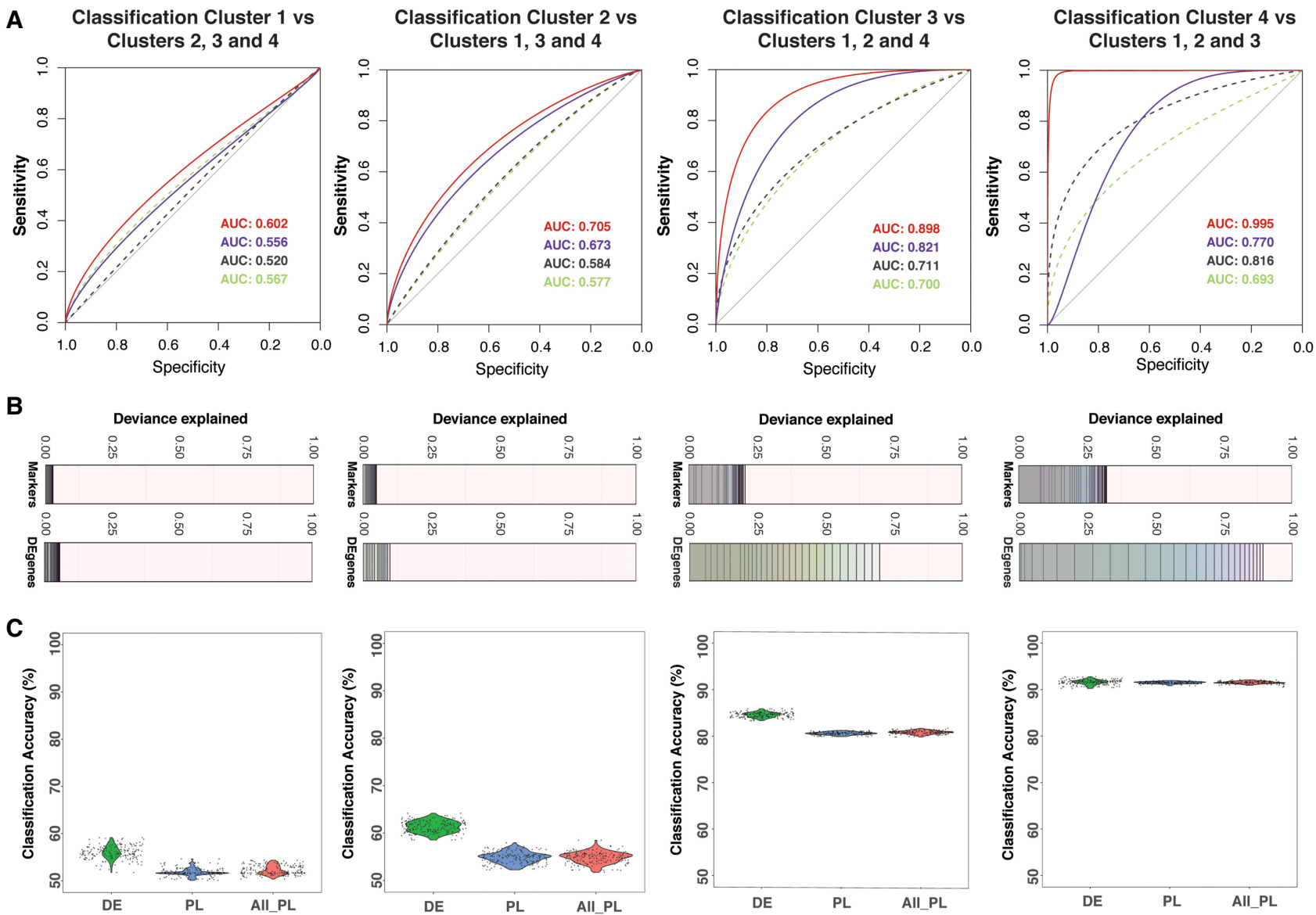

Figure 3. Selection of significant gene predictors for classifying each subpopulation using LASSO regression. (A) For each subpopulation, a LASSO model was run using a set of differentially expressed (DE) genes and another set of known markers. Dashed lines are receiver operating characteristic (ROC) curves for models using known markers. Continuous lines are for models using differentially expressed genes. The text shows corresponding area under the curve (AUC) values for ROC curves. For each case (known markers or DE genes), a model with the lowest AUC and another model with the highest AUC are given. Lower AUC values (and ROC curves) in the prediction models using known markers suggested that the models using DE genes performed better in sensitivity and specificity. (B) Each deviance plot shows the deviance explained ( $x$-axis) by a set of gene predictors (numbers of genes is shown as vertical lines and varies from 1 to maximum value as the total number of gene input or to the minimum number of genes that can explain most of the deviance). The remaining space between the last gene and 1.0 border represents deviance not explained by the genes in the model. (C) Classification accuracy calculated using a bootstrap method using all known markers (both pluripotent markers and primed lineage markers) or markers from our differentially expressed gene list is shown. Expression of LASSO-selected genes for subpopulation one and subpopulation two is shown in Supplemental Figure S7. The $x$-axis labels are for three cases: using LASSO-selected differentially expressed genes (DE); LASSO-selected pluripotency/lineage-primed markers (PL); and all pluripotency/lineage-primed markers (All PL).

cells within subpopulation two are located at the right terminal, indicating cells are closer to the root. As expected, cells within subpopulations three and four were located distal to cells from subpopulation two at the left terminal, which supports our results suggesting classification of subpopulations three and four as being more primed to differentiate. The diffusion algorithm, implemented through the Destiny package (Angerer et al. 2016), maps cells onto a two-dimensional pseudotime space. The results obtained from diffusion pseudotime analysis (Fig. 4C,D) were in strong agreement with the Monocle 2 prediction, supporting our evidence that the four subpopulations present in a culture of iPSCs exist in multiple states of pluripotency.

Validation against transcriptional data from the Human Induced Pluripotent Stem Cells Initiative (HipSci)

To confirm that genes selected by our LASSO analysis were also expressed in other hiPSC lines, we obtained open-access RNA-seq transcript count data (tags per million [TPM]) from the Human Induced Pluripotent Stem Cells Initiative (HipSci) for 71 hiPSC lines derived from reprogramming of dermal fibroblast biopsies from normal individuals (Streeter et al. 2017). Consistently, we observed expression of LASSO genes in 71 other independent hiPSC samples (Supplemental Fig. S8). Moreover, we observed high correlation $(r>0.85)$ between the relative expression values among genes in our single-cell data set with those genes in the HipSci bulk RNA-seq data set (Supplemental Fig. S8C). The high correlation further confirms that the single-cell sequencing data accurately reflects the relative abundance of transcripts.

\section{Transcriptional heterogeneity varies among cell} subpopulations

With the large-scale data from 18,787 single cells, more than 16,000 genes were detected as expressed. Using this expression matrix, we were able to robustly analyze expression heterogeneity, 


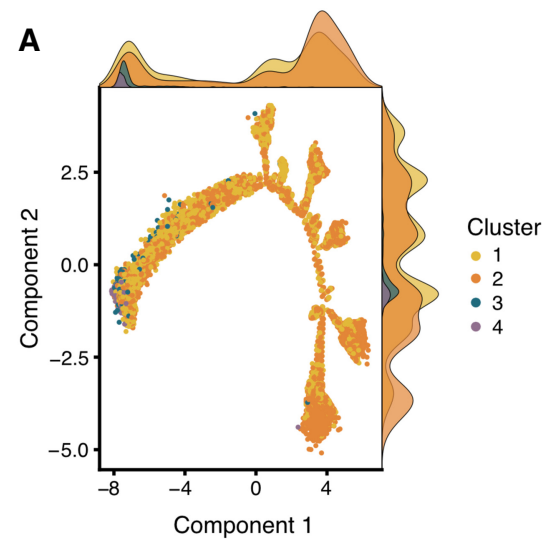

C

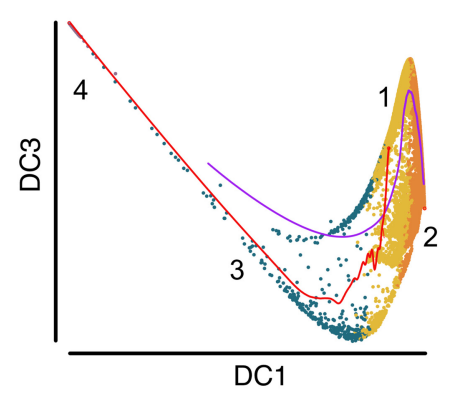

E

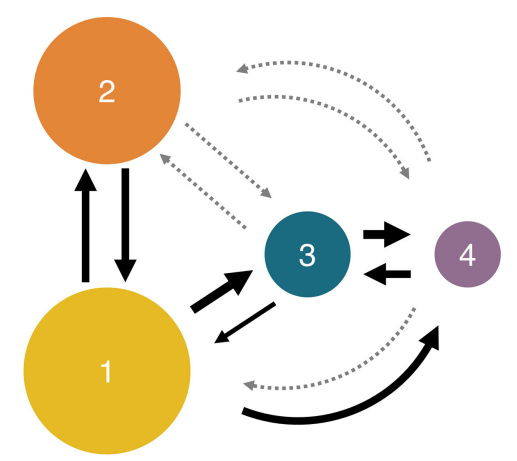

B

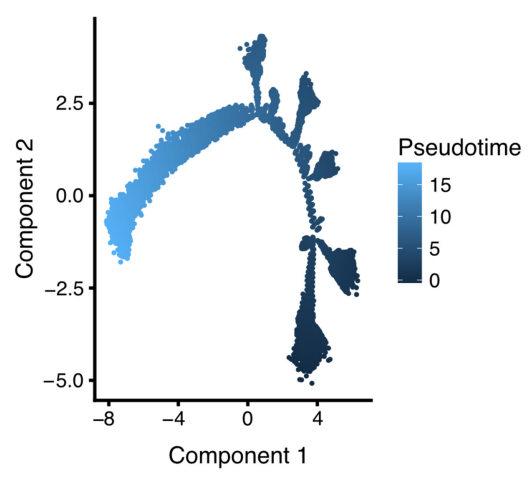

D

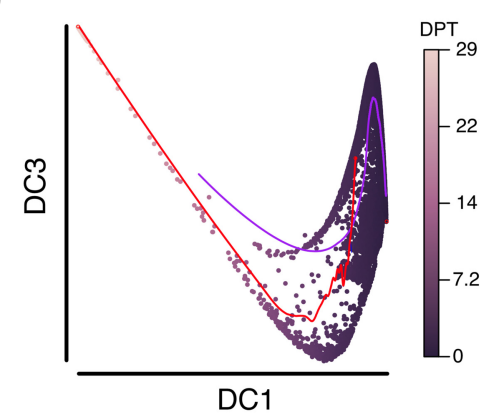

$\mathbf{F}$

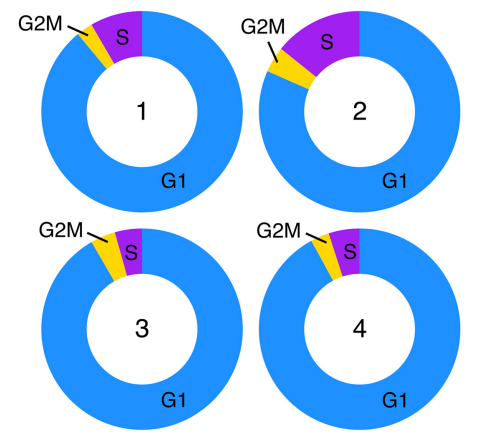

Figure 4. Trajectory and cell cycle membership analysis. Cell differentiation potential was mapped using two pseudotime approaches implemented in Monocle 2 and Destiny and a novel transition estimation method. $(A)$ The results of the Monocle 2 analysis, colored by subpopulation, and the normalized density of the cells in each location along the trajectory is shown as a density curve in the $x$ and $y$ plot margins. (B) The differentiation distance from the root cell to the terminal state, for which the dark blue represents the beginning (the root) and light blue represents the end (the most distant cells from the root) of the pseudotime differentiation pathway. $(C, D)$ The results of diffusion pseudotime analysis, colored by cluster $(C)$ and by diffusion pseudotime $(D)$. DC refers to diffusion component, and DPT refers to diffusion pseudotime. The red and blue pathways in $C$ and $D$ represent the transition path from cell to cell calculated by a random-walk algorithm. (E) We developed a novel approach that uses the LASSO classifier to quantify directional transitions between subpopulations. The percent of transitioning cells predicted between subpopulations. The weight of the arrows is relative to percentage (thicker is higher percentage), and the light gray dotted arrows represent percentages lower than 20. (F) Cell cycle stages were predicted for each cell by subpopulation. Subpopulation one ("Core") contains a significantly lower number of cells in the $S$ phase (synthesis) compared to subpopulation two ("Proliferative"; Fisher's exact test, $\left.P<2.2 \times 10^{-16}\right)$.

both between genes, and for a given gene, between cells and cell subpopulations (Supplemental Fig. S9). The inherently high heterogeneity of gene expression in scRNA-seq data, especially for low abundance genes with a more frequent on-off signal, may reduce the power to detect differential expression between cells (Shalek et al. 2013). Indeed, we identified more variation for sub-

populations with smaller numbers of cells (Supplemental Fig. S9A) and also for genes with low expression (Supplemental Fig. S9B). Tagwise dispersion, which is expression variability for a gene across all cells in a subpopulation, decreased when average expression increased (Supplemental Fig. S9B). The difference in the level of heterogeneity of gene expression for cells in a given subpopulation compared to other subpopulations is an important indicator of the relative dynamic cellular activity of the subpopulation. The red line in Supplemental Figure S9B shows the median dispersion of all genes across all cells within a subpopulation, thereby representing the average expression heterogeneity of the subpopulation. We found the median dispersion was higher in subpopulations three and four than in subpopulations one and two (Supplemental Fig. S9B). This is consistent with our conclusion that subpopulations three and four were closer to a differentiated state compared to cells in subpopulations one and two, which are identified as being more pluripotent based on transcriptome analysis.

\section{Cell cycle classification of hiPSC subpopulations}

To test the hypothesis that the subpopulations classified as core pluripotent (subpopulation one) or proliferative (subpopulation two) would harbor specific cell cycle signatures, we predicted the cell cycle phase of each cell (Scialdone et al. 2015; Lun et al. 2016). To estimate phase scores for each cell, the prediction method uses gene expression data and a reference training set containing prior ranks of relative expression of "marker pairs," in which the sign of each pair changes between cell cycle phases (Fig. 4F; Leng et al. 2015; Scialdone et al. 2015). In particular, we observed that a significantly higher percentage of cells in S (synthesis) phase belonged to subpopulation two (14.2\%) compared to subpopulation one $(8.4 \%$ : Fisher's exact test, $\left.P<2.2 \times 10^{-16}\right)$, and that a significantly higher percentage of cells in G1 (50.3\%) belonged to subpopulation one compared to subpopulation two (45.6\%: Fisher's exact test, $P<2.2 \times 10^{-16}$ ). Table 2 summarizes the proportions of cells in each cell cycle phase grouped by subpopulation. These data confirmed our previous findings that subpopulation two is more proliferative and likely forms the reversible, self-renewing component of the pluripotent population as a whole. 
Table 1. Percentage of cells in other subpopulations predicted by LASSO

\begin{tabular}{lcccc}
\hline & Subpop 1 & Subpop 2 & Subpop 3 & Subpop 4 \\
\hline Subpop 1 & - & 45.1 & 69.4 & 47.3 \\
Subpop 2 & 39.4 & $\overline{-}$ & 4.9 & 2.5 \\
Subpop 3 & 28.4 & 19.9 & - & 99.1 \\
Subpop 4 & 24.6 & 20.6 & 61.0 & - \\
\hline
\end{tabular}

\section{Discussion}

Although methods to dissect cell subpopulations at single-cell resolution such as FACS and immunohistochemistry have been available, a comprehensive profiling of transcriptional state(s) defining functionally distinct cell subpopulations composing a "homogenous" hiPSC cell line has not been described (Wilson et al. 2015; Kalkan et al. 2017). Although the heterogeneity of pluripotency states in an induced pluripotent cell culture is widely recognized, the quantitative characterization of subpopulations, identification of markers for subpopulations, and prediction of transition potential among them have not been investigated. To address this, we generated and analyzed the largest hiPSC single-cell transcriptomics data set to date, from five biological replicates of an engineered WTC-CRISPRi hiPSC line (Mandegar et al. 2016). The 18,787 highquality transcriptomes that compose this hiPSC population, collectively expressing 16,064 genes, provided strong statistical power for unbiased computational decomposition of cellular and transcriptional heterogeneity.

We developed a high-resolution and deterministic clustering method (UHRC) that does not require a predetermined cluster number. Across five separate biological replicates, we consistently found the existence of two main subpopulations, including a core pluripotent and a pluripotent-proliferative subpopulation, accounting for $96.1 \%$ of all cells profiled. Comparison of transcriptomes between subpopulations revealed a cell proliferation gene network, coordinately regulated by two transcription factors SALL4, ZIC1, and the NR5A1 signaling receptor, together with the well-characterized pluripotency regulators POU5F1, SOX2, and NANOG. Recently, Kalkan et al. (2017) detected higher expression of Sall4 and Nr5a2 by single-cell RNA-seq in the early undifferentiated state (2i) of mouse ESCs compared to later, more differentiated time points. The separation of two major subpopulations on the basis of cell proliferation states may in part be explained by evidence that reprogramming is commonly a stochastic process dependent on cell-proliferation rate (Hanna et al. 2009). It remains to be determined whether these subpopulations generally reflect a common feature of pluripotency in hESC or hiPSC populations. Furthermore, the high sensitivity of the UHRC method allowed the detection of two smaller subpopulations ( $2.8 \%$ and $1.1 \%$ of the total cells) with transcriptional signatures of pluripotency but primed for differentiation based on enriched signaling pathways and gene ontologies related to lineage specification. From analysis of expression heterogeneity within and between subpopulations, we found higher variability in these two subpopulations compared to the remaining cells. This observation is consistent with recent single-cell studies showing that the transition from pluripotency to lineage commitment phase is characterized by high gene expression variability (Semrau et al. 2017) and by the gradual destabilization of the pluripotent stem cell networks (Bargaje et al. 2017).

Moreover, we developed a method to find novel gene markers and models capable of distinguishing these subpopulations. Our marker identification method enables objective selection of genes from a large set containing hundreds to thousands of differentially expressed genes. The novel markers outperform known markers in predicting subpopulations. We developed a machine learning approach that can be widely applied to optimize prediction models based on single-cell transcriptomics data to classify cells into subpopulations at a high accuracy. Identifying cell types is often based on immunostaining, FACS, or targeted PCR quantification of a small number of markers (Tsankov et al. 2015; Kalkan et al. 2017). Here, we constructed an unbiased classification model based on differential gene expression selected by the LASSO penalized maximum likelihood optimization procedure. The procedure does not require model tuning by selecting parameters based on prior knowledge. We identified large, previously unreported groups of genes as new predictors for pluripotency and showed that prediction models from differentially expressed genes performed better than models built from known markers. Our model is especially effective at identifying genes and estimating their effects in the prediction of complex phenotypes (polygenic traits), which are controlled by multiple genetic loci and where each locus has a small individual effect (Yang et al. 2010a; Boyle et al. 2017). Our results support the use of an unbiased and transcriptome-wide approach to developing gene prediction models, which can leverage subtle expression changes in a large number of genes to accurately classify cell types (subpopulations) and discover novel gene

Table 2. Prediction of cell cycle phases for each of the 18,787 cells in four subpopulations

\begin{tabular}{|c|c|c|c|c|c|c|}
\hline Subpopulation ID & Phase & Cell number & Cluster percent & Cluster total & Phase percent & Phase total \\
\hline 1 & G1 & 8084 & $89^{* * *}$ & 9083 & 50.3 & 16,075 \\
\hline 2 & G1 & 7324 & 81.6 & 8977 & 45.6 & 16,075 \\
\hline 3 & G1 & 482 & 91.6 & 526 & 3 & 16,075 \\
\hline 4 & G1 & 185 & 92 & 201 & 1.2 & 16,075 \\
\hline 1 & G2M & 239 & 2.6 & 9083 & 37.3 & 640 \\
\hline 2 & G2M & 374 & 4.2 & 8977 & 58.4 & 640 \\
\hline 3 & G2M & 21 & 4 & 526 & 3.3 & 640 \\
\hline 4 & G2M & 6 & 3 & 201 & 0.9 & 640 \\
\hline 1 & $S$ & 760 & 8.4 & 9083 & 36.7 & 2072 \\
\hline 2 & $S$ & 1279 & $14.2^{* * *}$ & 8977 & 61.7 & 2072 \\
\hline 3 & $S$ & 23 & 4.4 & 526 & 1.1 & 2072 \\
\hline 4 & $S$ & 10 & 5 & 201 & 0.5 & 2072 \\
\hline
\end{tabular}

${ }^{(* * *)}$ Cluster 1 versus $2: P<2.2 \times 10^{-16}$, Fisher's exact test. 
markers for important phenotypes. The results of these predictions can generate important hypotheses around genes and networks to be validated by functional genomics assays.

Importantly, using LTS, our quantitative and directional prediction method, we observed that these multiple iPSC subpopulations have the potential to transition between pluripotency states, which was further supported by two independent analysis methods: Monocle 2 and diffusion pseudotime. The results of these trajectory analyses revealed the dynamic transition among subpopulations that ensures both cell state renewal (transition between subpopulation two and subpopulation one) and differentiation capacity (subpopulation one to subpopulations three and four). This is especially the case for subpopulations one and two, which are on a continuum yet are distinguishable from one another based on their transcriptional profiles. However, between subpopulations two and three and subpopulations two and four, the continuum is less clear, suggesting greater distinction between each subpopulation (Fig. 4). Prior work using microarray gene expression arrays on human embryonic stem cells (hESCs) suggested a continuum of pluripotent states exists (Hough et al. 2009). Our finding is consistent with a study on early developmental progression from naive pluripotency in human epiblast and embryonic stem cells to more differentiated tissues such as trophectoderm and primitive endoderm (Yan et al. 2013). Using single cells, the authors reported a strong reduction in the expression of pluripotency markers during the cell transition from pluripotent to differentiated. A second study investigating early developmental progression from naive pluripotency in mouse ESCs (Kalkan et al. 2017) showed reduced expression of pluripotency markers occurred prior to the activation of lineage specification markers.

Despite the large number of cells sequenced, this study was limited in that only $3^{\prime}$ mRNA was sequenced; thus there remained variation between cell populations that could not be detected. In addition, our observations were obtained from one iPSC line and may not reflect the general behavior of all pluripotent stem cells. Nevertheless, our aim was to deconvolute a "homogeneous" hiPSC population, and inclusion of transcriptional sequence data from other RNA species in the future will likely improve our ability to further delineate subpopulations of cells. Furthermore, we confirmed that the genes selected were expressed in 71 HipSci data sets (Streeter et al. 2017), and the relative expression level among genes was consistent between scRNA and bulk RNA sequencing. The parental cell line selected for this study, WTCCRISPRi hiPSCs (Mandegar et al. 2016), is an important system for targeted transcription inhibition and is a key feature for functional genomics studies that build on this data set to study the biology of pluripotency. Coupled with high-throughput single-cell RNA-seq, our innovative computational methods have revealed the intrinsic characteristics that distinguish subpopulations of pluripotent stem cells. Future work is required to expand this analysis to multiple hiPSC and hESC lines to identify common features of single-cell subpopulations in pluripotency.

\section{Methods}

\section{Cell culture}

Undifferentiated human induced pluripotent stem cells (hiPSC; wild type C [WTC]) were provided courtesy of Bruce Conklin (UCSF and Gladstone Institutes) as previously described (Mandegar et al. 2016). Cells were maintained on Vitronectin XF (STEMCELL Technologies, catalog no. 07180) and cultured in mTeSR1
(STEMCELL Technologies, catalog no. 05850). Cytogenetic analysis by Giemsa banding showed a normal 46, XY male karyotype. For scRNA-seq, samples 1 and 2 were harvested from a single plate using Versene, split into two technical replicates, resuspended in Dulbecco's PBS (dPBS; Life Technologies, catalog no. 14190-144) with $0.04 \%$ bovine serum albumin (Sigma, catalog no. A9418$50 \mathrm{G})$, and immediately transported for cell sorting. For samples $3-5$, cells were harvested from individual plates using $0.25 \%$ Trypsin (Life Technologies, catalog no. 15090-046) in Versene, neutralized using 50\% fetal bovine serum (HyClone, catalog no. SH30396.03) in DMEM/F12 (Life Technologies, catalog no. 11320-033), centrifuged at $1200 \mathrm{rpm}$ for $5 \mathrm{~min}$, and resuspended in $\mathrm{dPBS}+0.04 \% \mathrm{BSA}$.

\section{Cell sorting}

Viable cells were sorted on a BD Influx cell sorter (BectonDickinson) using Propidium Iodide into Dulbecco's PBS + 0.04\% bovine serum albumin and retained on ice. Sorted cells were counted and assessed for viability with Trypan Blue using a Countess automated counter (Invitrogen) and then resuspended at a concentration of $800-1000$ cells $/ \mu \mathrm{L}\left(8 \times 10^{5}-1 \times 10^{6}\right.$ cells/ $\mathrm{mL}$ ). Final cell viability estimates ranged between $80 \%$ and $93 \%$.

\section{Generation of single-cell GEMs and sequencing libraries}

Single-cell suspensions were loaded onto 10x Genomics Single Cell 3' Chips along with the reverse transcription (RT) mastermix per the manufacturer's protocol for the Chromium Single Cell 3' Library (10x Genomics; PN-120233) to generate single-cell gel beads in emulsion (GEMs). Reverse transcription was performed using a C1000 Touch Thermal Cycler with a Deep Well Reaction Module (Bio-Rad) as follows: for $2 \mathrm{~h}$ at $55^{\circ} \mathrm{C}$; for $5 \mathrm{~min}$ at $85^{\circ} \mathrm{C}$; hold $4^{\circ} \mathrm{C}$. cDNA was recovered and purified with DynaBeads MyOne Silane Beads (Thermo Fisher Scientific; catalog no. 37002D) and SPRIselect beads (Beckman Coulter; catalog no. B23318). Purified cDNA was amplified as follows: for $3 \mathrm{~min}$ at $98^{\circ} \mathrm{C}$; $12 \times$ (for $15 \mathrm{sec}$ at $98^{\circ} \mathrm{C}$; for $20 \mathrm{sec}$ at $67^{\circ} \mathrm{C}$; for $60 \mathrm{sec}$ at $72^{\circ} \mathrm{C}$ ); for $60 \mathrm{sec}$ at $72^{\circ} \mathrm{C}$; hold $4^{\circ} \mathrm{C}$. Amplified cDNA was purified using SPRIselect beads and sheared to $\sim 200 \mathrm{bp}$ with a Covaris S2 instrument (Covaris) using the manufacturer's recommended parameters. Sequencing libraries were generated with unique sample indices (SI) for each sample. Libraries for samples 1-3 and 4-5 were multiplexed, respectively, and sequenced on an Illumina NextSeq 500 (NextSeq control software v2.0.2/Real Time Analysis v2.4.11) using a 150-cycle NextSeq 500/550 High Output Reagent Kit v2 (Illumina, FC-404-2002) in standalone mode as follows: $98 \mathrm{bp}$ (Read 1), $14 \mathrm{bp}$ (I7 Index), 8 bp (I5 Index), and $10 \mathrm{bp}$ (Read 2).

\section{Bioinformatics mapping of reads to original transcripts and cells}

Processing of the sequencing data into transcript count tables was performed using the Cell Ranger Single Cell Software Suite 1.2.0 by 10x Genomics (https://www.10xgenomics.com/). Raw base call files from the NextSeq 500 sequencer were demultiplexed, using the cellranger mkfastq pipeline, into sample-specific FASTQ files. These FASTQ files were then processed with the cellranger count pipeline where each sample was processed independently. First, cellranger count used STAR (Dobin et al. 2013) to align cDNA reads to the hg19 human reference transcriptome, which accompanied the Cell Ranger Single Cell Software Suite 1.2.0. We note that, because the expression data is limited to the $3^{\prime}$ end of a gene and we used gene-level annotations, differences between reference versions, such as GRCh38, are unlikely to significantly alter conclusions. Aligned reads were filtered for valid cell barcodes and 
unique molecular identifiers (UMI), and observed cell barcodes were retained if they were 1-Hamming-distance away from an entry in a whitelist of known barcodes. UMIs were retained if they were not homopolymers and had a quality score $>10$ (90\% base accuracy). cellranger count corrected mismatched barcodes if the base mismatch was due to sequencing error, determined by the quality of the mismatched base pair and the overall distribution of barcode counts. A UMI was corrected to another, more prolific UMI if it was 1-Hamming-distance away and it shared the same cell barcode and gene. cellranger count examined the distribution of UMI counts for each unique cell barcode in the sample and selected cell barcodes with UMI counts that fell within the 99th percentile of the range defined by the estimated cell count value. The default estimated cell count value of 3000 was used for this experiment. Counts that fell within an order of magnitude of the 99th percentile were also retained. The resulting analysis files for each sample were then aggregated using the cellranger aggr pipeline, which performed a between-sample normalization step and merged all five samples into one. Post-aggregation, the count data was processed and analyzed using a comprehensive pipeline, assembled and optimized in-house as described below.

\section{Preprocessing}

To preprocess the mapped data, we constructed a cell-quality matrix based on the following data types: library size (total mapped reads), total number of genes detected, percent of reads mapped to mitochondrial genes, and percent of reads mapped to ribosomal genes. Cells that had any of the four parameter measurements higher than $3 \times$ median absolute deviation (MAD) of all cells were considered outliers and removed from subsequent analysis (Supplemental Table S2). In addition, we applied two thresholds to remove cells with mitochondrial reads $>20 \%$ or ribosomal reads $>50 \%$ (Supplemental Table S2). To exclude genes that were potentially detected from random noise, we removed genes that were detected in fewer than $1 \%$ of all cells. Before normalization, abundantly expressed ribosomal protein genes and mitochondrial genes were discarded to minimize the influence of those genes in driving clustering and differential expression analysis.

\section{Data normalization}

Two levels of normalization were performed to reduce possible systematic bias between samples and between cells. To reduce potential confounding effects caused by differences in sequencing depths among the five samples, a subsampling process (Zheng et al. 2017) was used to scale the mean mapped reads (MMR) per cell of all samples down to the level of the sample with the lowest MMR. For each sample, a binomial sampling process randomly selected reads and UMIs for each gene in a cell at a sample-specific subsampling rate. The subsampling rate for each sample was determined using the ratios of expected total reads (given the expected mean reads per cell [minimum MMR of all samples], the known number of cells, and the fraction of mapped reads to total reads) to the original total mapped reads (equation 1). Following resampling, the MMRs for the five samples were scaled, and the expression data distribution for genes in all cells of the sample was for genes in all cells of the sample was maintained.

$$
\text { Rate }_{i}=\min \left(\mathrm{MMR}_{j}\right) * N_{i} * \frac{\left(\text { ReadFraction }_{i}\right)}{\text { Total_mapped_reads }_{i}},
$$

where $\min \left(\mathrm{MMR}_{\mathrm{j}}\right)$ is the minimum $\mathrm{MMR}$ of all samples to be merged; $N_{j}$ is the number of cells in sample $j$; ReadFraction $n_{\mathrm{i}}$ is the ratio of confidently mapped reads in a cell to the total number of reads detected for that cell in sample $i$; and Total_mapped_reads $s_{\mathrm{i}}$ is the total number reads that share the same cell barcode. For each gene in each cell, we performed a random binomial sampling process of reads with the probability equal the subsample rate calculated in equation 1 . This process is more robust than standard scaling options because it takes into account unique read information associated with mapped genes and cells.

To reduce cell-specific bias, possibly caused by technical variation (such as cDNA synthesis, PCR amplification efficiency, and sequencing depth for each cell), expression values for all genes in a cell were scaled based on an estimated cell-specific size factor. Before normalization, counts were $\log _{2}$-transformed (by $\log _{2}($ count +1$\left.)\right)$ to stabilize variance due to the large range of count values (spanning six orders of magnitude) (Supplemental Fig. S1E). To estimate the scaling size factor for each cell, a deconvolution method (Lun et al. 2016) was applied for summation of gene expression in groups of cells. This summation approach reduced the number of stochastic zero expression of genes that are lowly expressed (higher dropout rates), or genes that are turned on/off in different subpopulations of cells.

$$
E\left(V_{i k}\right)=\lambda_{i 0} \sum_{j \in S_{k}} \theta_{j} t_{j}^{-1},
$$

where $S_{k}$ is a pool of cells; $V_{i k}$ is the sum of adjusted expression value $\left(Z_{i j}=\theta_{j}^{*} \lambda_{i 0}\right.$, where $\lambda_{i 0}$ is the expected transcript count and $\theta_{j}$ is the cell-specific bias) across all cells in pool $V_{k}$ for gene $i$; and $\theta_{j} t_{j}^{-1}$ is the cell-specific scale factor for cell $j$ (where $t_{j}$ is the constant adjustment factor for cell $j$ ).

The estimated size factor of a gene in pool $S_{k}$, named as $E\left(R_{i k}\right)$, is the ratio between the estimated $V_{i k}$ and the average $Z_{i j}$ across all cells in the population. Therefore, $E\left(R_{i k}\right) \approx \sum_{s_{k}} \theta_{j} t_{j}^{-1} C^{-1}$ where $C=N^{-1} \sum_{s_{0}} \theta_{j} t_{j}^{-1}$, and where $N$ is the number of cells; $S_{0}$ represents all cells and is a constant for the whole population and thus can be set to unity or ignored. The cell pools were sampled using a sliding window on a list of cells ranked by library size for each cell. Four sliding windows with $20,40,60$, and 80 cells were independently applied, and the results were combined to generate a linear system that can be decomposed by QR decomposition to estimate $\theta_{j} t_{j}^{-1}$ size factor for each of the cell. The normalized counts are the results of taking the raw counts divided by cell-specific normalized size factors.

\section{Developing a publicly accessible data resource}

To make this valuable single-cell human pluripotent singlecell data set publicly accessible, we created an interactive R Shiny server at http://computationalgenomics.com.au/shiny/hipsc/. The server contains user-friendly data exploration and representation tools. Users can interactively explore the expression of any selected gene from the 16,064 genes in each cell of the 18,787 cells. The data specific for each subpopulation are also calculated. For each gene, a summary table, t-SNE plot, and a density plot can be generated, and the results are downloadable.

\section{Analyzing transcriptional heterogeneity in a population of single cells}

To assess transcriptional heterogeneity among cells and genes, we first removed potential variation due to technical sources by the subsampling process and the cell-specific normalization as described above. Depending on experimental design, an additional step using a generalized linear model (GLM) to regress out other potential confounding factors can be included. After reducing technical variation via normalization, we calculated the coefficient of variation and expression dispersion of each gene across all cells. For cell-to-cell variation, we first performed principal

\section{Genome Research}

www.genome.org 
component analysis (PCA) on general cell data, which included percent counts of the top 100 genes, total number of genes, and percent of mitochondrial and ribosomal genes. To investigate variation between genes, the distribution of dispersion across a range of expression values was calculated (equation 3 ). This approach is useful because technical variation often appears greater in lowly expressed genes than in more abundant genes (Shalek et al. 2013). Denoting $x_{i}$ as the vector of expression values (in counts per million) for gene $i$ across all cells, we used the following formula to compute coefficient of variation $(c v)$ :

$$
c v=\sqrt{\frac{\operatorname{var}\left(x_{i}\right)}{\operatorname{mean}\left(x_{i}\right)}} .
$$

We estimated the biological coefficient of variation (BCV) with an empirical Bayesian approach to estimate dispersion between genes and between samples (McCarthy et al. 2012). Common dispersion (shared dispersion value of all genes), trended dispersion (mean dispersion trend for lowly expressed genes to abundant genes), and gene-specific dispersion was estimated to reflect variation of all genes across the whole population (Supplemental Fig. S9).

\section{Dimensionality reduction}

After merging five samples, preprocessing, and normalizing the data set was scaled to Z-distribution, and PCA was performed for dimensionality reduction using the prcomp function in $\mathrm{R}$ (McCarthy et al. 2017). To assess PCA results, we examined the top genes that were most correlated to PC1 and PC2 and the distribution of cells and percent variance explained by the top five PCs. Importantly, the optimal number of PCs explaining the most variance in the data set was determined using a Scree test calculated by the fa.parallel function in the psych package. The fa.parallel was run based on expression data for the top variable genes.

Cells are represented using t-SNE and diffusion map (van der Maaten and Hinton 2008). We used the Rtsne package v0.1.3 with the normalized expression data (16,064 genes by 18,787 cells) to calculate a three-dimensional t-SNE projection data set $(16,064$ genes by three t-SNE dimensions), which was then combined with other data types to display cells on two- and three-dimensional t-SNE plots.

\section{Clustering}

We developed an unsupervised clustering method that does not require the number of clusters to be specified prior to the analysis so that small and big clusters can be determined automatically. We first computed a cell-PCA eigenvector matrix based on the full expression data set (all 18,787 cells and over 15,953 genes) to reduce the full data set to a 18,787 cells $\times 10$ first orthogonal principal components (PCs). The first 10 PCs that accounted for most of the variance of the full expression matrix were used for computing cell-to-cell Euclidean distance. We applied an agglomerative hierarchical clustering (HAC) procedure using Ward's minimum variance (minimal increase of sum of squared) method aiming at finding compact clusters to minimize total within-cluster variance. By using Ward's linkage method, cells and branches are joined iteratively from the bottom (each cell is one branch) to the top (all cells form one cluster), resulting in a complete dendrogram tree. To consider the broadest possible solution space for finding clusters from the tree without a constraining threshold, we allowed a cut height of $99 \%$ of the range between the 5 th percentile and the maximum of the joining height on the dendrogram, allowing the entire set of branches in the original tree to be considered in the cluster search space.
To determine the number of subpopulations, branches of the dendrogram were pruned by a Dynamic Tree Cut method, which uses an approach that does not attempt to merge all branches with distance lower than a constant (supervised) height cutoff (Langfelder et al. 2008). The dynamic merging process is a hybrid method that uses the dendrogram tree shape to define the bottom level clusters (based on number of cells, distance between cells, core of the branches, and gaps between branches) and uses the dissimilarity measure to merge cells/branches (with lowest dissimilarity) into the initially defined clusters. The dynamic, iterative decomposition and combination of clusters enables the sensitive detection of outliers and nested clusters. To ascertain the clustering results are stable, we performed cluster stability analysis and resolution analysis. We narrowed the clustering search space by running 10 clustering iterations at the bottom $25 \%$ height of the entire tree. In each iteration, we decreased the pruning window by $2.5 \%$. The bottom $25 \%$ of the tree was used in the stability analysis to keep clusters at the highest resolution, allowing detection of small clusters. Among all 10 independent clustering iterations, we consistently found four clusters (Supplemental Fig. S4).

To allow for the detection of the smallest subpopulation, we considered any cluster with more than 20 cells to be significant in size, thereby enabling the detection of a cluster as small as $0.1 \%$ of the total cells. With these two parameters, the whole tree and the smallest clusters are considered without predetermined parameters.

Cluster information was then overlaid on cells in two- to three-dimensional t-SNE plots. Notably, the clusters were the results from HAC analysis and were not dependent on the t-SNE plots, which were used purely for representation purposes.

\section{Cell cycle analysis}

To assess whether the clustering assignments were affected by the differences in cell cycle phases, we applied a machine learning prediction model to predict cell cycle phase (Scialdone et al. 2015; Lun et al. 2016). The model uses scRNA gene expression data and a reference training set (prior knowledge) on relative expression of "marker pairs," in which the sign of each pair changes between cell cycle phases (Scialdone et al. 2015). Scores for each of the three phases (G1, G2M, and S) were estimated based on the proportion of training pairs having the sign changed in each phase relative to the other phases. The human training set was from Leng et al. (2015).

\section{Differential expression analysis}

To select genes that distinguish subpopulations, we performed pairwise differential expression analysis between cells in pairs or groups of clusters by fitting a general linear model and using a negative binomial test as described in the DESeq package (Anders and Huber 2010). Each cell was considered as one biological replicate in each cluster. We found that the shrinkage estimation of dispersion approach used in DESeq produced stable estimation of scale factors for genes and cells between clusters and was more conservative in detecting differentially expressed genes, especially when comparing subpopulations with a larger number of cells (such as subpopulation one and two) to subpopulations with small cell numbers (such as subpopulations three and four). Specifically, DESeq detected fewer DE genes that expressed highly in a small proportion of cells in a subpopulation, whereas remaining cells in that subpopulation had zero or very low expression. Significantly differentiated genes were those with Bonferroni threshold $P$-values $<5 \%$ $\left(P<3.1 \times 10^{-7}\right)$. 


\section{Subpopulation classification analysis}

To develop predictive models based on single-cell transcriptomics data, we applied the Least Absolute Shrinkage and Selection Operator (LASSO) procedure (Tibshirani 1996). LASSO selects gene predictors for classifying cells into one of the four subpopulations. Briefly, penalized logistic regression was applied to fit a predictor matrix (training set) containing expression values of the top differentially expressed genes in 50\% of the total cells-or for the smallest subpopulation four, a subsample of a randomly selected $10 \%$ of the total cells-and a response variable vector assigning cells into one of the subpopulations (dichotomous variable, with two classes: belonging to the subpopulation or belonging to the remaining cells). The LASSO procedure optimizes the combination set of coefficients for all predictors in a way that the residual sum of squares is smallest for a given lambda value (Friedman et al. 2010).

We applied the glmnet $\mathrm{R}$ package to estimate parameters by a penalized maximum likelihood procedure (as shown in equation 4 ) so that insignificant genes $j$ (not explaining variance) were removed (coefficients shrunken to 0 ):

$$
\operatorname{Minimize}\left(\frac{1}{N} \sum_{i=1}^{n} l\left(y_{i}, \beta_{0}+\sum_{j=1}^{p} \beta_{j} x_{i j}\right)+\lambda \sum_{j=1}^{p}\left|\beta_{j}\right|\right),
$$

where $\mathbf{x}_{\mathbf{i}}=\left(\mathrm{x}_{i 1}, \mathrm{x}_{i 2}, \ldots, \mathrm{x}_{i \mathrm{p}}\right)$ is a vector of expression values of $p$ genes in cell $\mathrm{C}_{i} ; y_{i}$ is the cell subpopulation class of the cell $\mathrm{C}_{i}$; $l\left(y_{i}, \beta_{0}+\sum_{j=1}^{p} \beta_{j} x_{i j}\right)$ is the negative $\log$-likelihood for $C_{i}$; and $\lambda$ is a tuning parameter that controls the shrinkage penalty of the coefficients.

For each training data set, an optimal $\lambda$ and a set of gene predictors can be determined by a 10 -fold cross-validation procedure to select the $\lambda$ that produced the minimum classification errors. The LASSO procedure optimizes the combination set of coefficients for all predictors in a way that the residual sum of squares is smallest for a given lambda value (Friedman et al. 2010). In other words, the LASSO procedure identified an optimal combination of genes (predictors) and fitted a logistic regression model. The logistic regression model is a generalized linear model in which the response variable belongs to the binomial family, in which expression values of the selected genes were predictors, and the binary labels (classes) of cells were response variables. The fitted model could either explain the highest deviance, compared to the full model, or classify cells to subpopulations with the lowest 10 -fold classification error. The glmnet $\mathrm{R}$ package was applied to select top genes from the differentially expressed genes that contributed to classifying cells into each subpopulation (Tibshirani et al. 2012). To calculate the model prediction accuracy, we trained the LASSO model using one subsampled data set and then evaluated the trained model in predicting a new, non-overlapping subsampled data set. By comparing the prediction results with the known subpopulation label of each cell, the model prediction accuracy was calculated. We applied a bootstrap procedure to calculate classification accuracy for 100 iterations.

\section{Pseudotime analysis}

We applied the optimal LASSO classification model trained in the procedure above to estimate transition scores between subpopulation. For two subpopulations, the transition score is the percentage of cells in the target subpopulation that are classified as belonging to the original subpopulation and not belonging to the other class. For each subpopulation, the logistic regression model (with LASSO-selected genes and corresponding coefficients) was fitted into a new expression data set for all cells in an- other subpopulation. The model estimates the conditional class probability of a cell $C_{i}$ belonging to a class $k$ ( 0 or $1 \equiv$ belong or not belong to) given the gene expression profile $\mathbf{x}_{\boldsymbol{i}}$ of the $p$ LASSO-selected genes $\mathbf{x}_{\boldsymbol{i}}$.

The conditional class probabilities of cell $C_{i}$ belonging to class $k$ is then the linear combination of selected genes, and can be estimated as:

$$
\begin{aligned}
\ln (\operatorname{Pr}(y=1 \mid X=x)) & =\beta_{0}+\beta_{1} x_{1}+\beta_{2} x_{2}+\cdots+\beta_{p} x_{P} \\
& =\beta_{0}+\mathbf{x} \beta^{\prime},
\end{aligned}
$$

where $\beta_{j}$ is a coefficient for gene $j\left(\beta_{j}=0\right.$ if the gene $j$ is not a predictor of the class). The coefficient vector $\beta=\left(\beta_{0}, \beta_{1}, \ldots \beta_{p}\right)$ can be calculated by maximum likelihood estimation and the deviance that best explains the variance compared to the full model, as in equation 4 . The predicted probability of a cell $C_{i}$ being in a subpopulation one or zero is estimated by replacing $\beta$ and gene expression values in the regression equation 5 .

Results from our novel trajectory analysis method was compared to the two state-of-the-art pseudotime analysis approaches, namely Diffusion pseudotime (Haghverdi et al. 2016) and Monocle 2 (Qiu et al. 2017). The Diffusion method, implemented in Destiny (version 2.0.8), applies a diffusion-like random-walk algorithm to estimate the cell's probabilities, based on a weighted nearest neighbor graph, of transitioning into another cell at a different fate/state, thereby inferring a differentiation trajectory. The diffusion approach is not dependent on dimension reduction. The Monocle 2 package (version 2.2.0) applies an unsupervised manifold learning technique, namely reverse graph embedding (Discriminative Dimensionality Reduction for learning principal graphs [DDRTree]), to learn an optimal path (a curved manifold in a low dimensional space) that approximates the structure of high-dimensional data and reversely map cells on this path back to the original multidimensional space so that nearby cells on the manifold are also nearby cells on the original space (Qiu et al. 2017). Both the Monocle 2 and Diffusion methods estimate pseudotime, which is a measure of how far a cell progresses in the differentiation process compared to a root cell. Pseudotime is therefore the distance between two cells in a modeled trajectory and is estimated independently from the experimental time.

\section{Pathway and gene functional analysis}

To functionally characterize the four subpopulations, we performed a network analysis using significant DE genes between cells within a subpopulation and the remaining cells, or between cells in pairs of subpopulations. We used Cytoscape to apply three main programs: GeneMANIA (Warde-Farley et al. 2010), with a comprehensive background database containing 269 networks and 14.3 million interactions; the Reactome functional interaction network analysis, a reliably curated protein functional network (Wu et al. 2010); and the STRING protein-protein interaction database (Szklarczyk et al. 2015).

\section{Data access}

The raw and processed data from this study have been submitted to ArrayExpress (https://www.ebi.ac.uk/arrayexpress/) under accession number E-MTAB-6687. All code for figures and analysis are available as Supplemental_Code.zip and at GitHub (https:// github.com/IMB-Computational-Genomics-Lab/hiPSC_paper_ 2018).

\section{Genome Research}

www.genome.org 


\section{Acknowledgments}

Sequencing was performed by the Institute for Molecular Bioscience Sequencing Facility at the University of Queensland. We thank Stacey Andersen for valuable discussions and Liam Crowhurst for contributing to the development of the interactive Shiny apps. This work was supported by the Australian National Health and Medical Research Council (NHMRC) grants APP1083405 and APP1107599.

Author contributions: J.E.P. designed the study, acquired funding, and led analysis. N.J.P. led the cell culture and informed on the interpretation of results. S.W.L., H.S.C., T.J.C.B., and A.N.C. performed molecular experiments. Q.H.N., S.W.L., and A.S. performed analysis. All authors wrote and edited the manuscript.

\section{References}

Anders S, Huber W. 2010. Differential expression analysis for sequence count data. Genome Biol 11: R106.

Angerer P, Haghverdi L, Büttner M, Theis FJ, Marr C, Buettner F. 2016. destiny: diffusion maps for large-scale single-cell data in R. Bioinformatics 32: $1241-1243$.

Artavanis-Tsakonas S, Rand MD, Lake RJ. 1999. Notch signaling: cell fate control and signal integration in development. Science 284: 770-776.

Bargaje R, Trachana K, Shelton MN, McGinnis CS, Zhou JX, Chadick C, Cook S, Cavanaugh C, Huang S, Hood L. 2017. Cell population structure prior to bifurcation predicts efficiency of directed differentiation in human induced pluripotent cells. Proc Natl Acad Sci 114: 22712276.

Boyer LA, Lee TI, Cole MF, Johnstone SE, Levine SS, Zucker JP, Guenther MG, Kumar RM, Murray HL, Jenner RG, et al. 2005. Core transcriptional regulatory circuitry in human embryonic stem cells. Cell 122: 947-956.

Boyle EA, Li YI, Pritchard JK. 2017. An expanded view of complex traits: from polygenic to omnigenic. Cell 169: 1177-1186.

Dobin A, Davis CA, Schlesinger F, Drenkow J, Zaleski C, Jha S, Batut P, Chaisson M, Gingeras TR. 2013. STAR: ultrafast universal RNA-seq aligner. Bioinformatics 29: 15-21.

Evans MJ, Kaufman MH. 1981. Establishment in culture of pluripotential cells from mouse embryos. Nature 292: 154-156.

Forristal CE, Wright KL, Hanley NA, Oreffo RO, Houghton FD. 2010. Hypoxia inducible factors regulate pluripotency and proliferation in human embryonic stem cells cultured at reduced oxygen tensions Reproduction 139: 85-97.

Friedman JH, Hastie T, Tibshirani R. 2010. Regularization paths for generalized linear models via coordinate descent. I Stat Softw 33: $1-22$.

Fuhrmann G, Chung ACK, Jackson KJ, Hummelke G, Baniahmad A, Sutter J, Sylvester I, Schöler HR, Cooney AJ. 2001. Mouse germline restriction of Oct4 expression by germ cell nuclear factor. Dev Cell 1: 377-387.

Guenther MG. 2011. Transcriptional control of embryonic and induced pluripotent stem cells. Epigenomics 3: 323-343.

Haghverdi L, Buttner M, Wolf FA, Buettner F, Theis FJ. 2016. Diffusion pseudotime robustly reconstructs lineage branching. Nat Methods 13: 845-848.

Hanna J, Saha K, Pando B, van Zon J, Lengner CJ, Creyghton MP, van Oudenaarden A, Jaenisch R. 2009. Direct cell reprogramming is a stochastic process amenable to acceleration. Nature 462: 595-601.

Hough SR, Laslett AL, Grimmond SB, Kolle G, Pera MF. 2009. A continuum of cell states spans pluripotency and lineage commitment in human embryonic stem cells. PLoS One 4: e7708.

Inoue T, Ota M, Ogawa M, Mikoshiba K, Aruga J. 2007. Zic1 and Zic3 regulate medial forebrain development through expansion of neuronal progenitors. J Neurosci 27: 5461-5473.

Kalkan T, Olova N, Roode M, Mulas C, Lee HJ, Nett I, Marks H, Walker R, Stunnenberg HG, Lilley KS, et al. 2017. Tracking the embryonic stem cell transition from ground state pluripotency. Development 144: 1221-1234.

Lam CS, Mistri TK, Foo YH, Sudhaharan T, Gan HT, Rodda D, Lim LH, Chou C, Robson P, Wohland T, et al. 2012. DNA-dependent Oct4Sox 2 interaction and diffusion properties characteristic of the pluripotent cell state revealed by fluorescence spectroscopy. Biochem J 448: 21-33.

Langfelder P, Zhang B, Horvath S. 2008. Defining clusters from a hierarchical cluster tree: the Dynamic Tree Cut package for R. Bioinformatics 24: 719-720.
Leng N, Chu LF, Barry C, Li Y, Choi J, Li X, Jiang P, Stewart RM, Thomson JA, Kendziorski C. 2015. Oscope identifies oscillatory genes in unsynchronized single-cell RNA-seq experiments. Nat Methods 12: 947950.

LeVincent SD, Dunn NR, Hayashi S, Norris DP, Robertson EJ. 2003. Cell fate decisions within the mouse organizer are governed by graded Nodal signals. Genes Dev 17: 1646-1662.

Lun AT, Bach K, Marioni JC. 2016. Pooling across cells to normalize singlecell RNA sequencing data with many zero counts. Genome Biol 17: 75.

Maere S, Heymans K, Kuiper M. 2005. BiNGO: a Cytoscape plugin to assess overrepresentation of Gene Ontology categories in Biological Networks. Bioinformatics 21: 3448-3449.

Mandegar MA, Huebsch N, Frolov EB, Shin E, Truong A, Olvera MP, Chan AH, Miyaoka Y, Holmes K, Spencer CI, et al. 2016. CRISPR interference efficiently induces specific and reversible gene silencing in human iPSCs. Cell Stem Cell 18: 541-553.

McCarthy DJ, Chen Y, Smyth GK. 2012. Differential expression analysis of multifactor RNA-seq experiments with respect to biological variation. Nucleic Acids Res 40: 4288-4297.

McCarthy DJ, Campbell KR, Lun AT, Wills QF. 2017. Scater: pre-processing, quality control, normalization and visualization of single-cell RNA-seq data in R. Bioinformatics 33: 1179-1186.

Miller A, Ralser M, Kloet SL, Loos R, Nishinakamura R, Bertone P, Vermeulen M, Hendrich B. 2016. Sall4 controls differentiation of pluripotent cells independently of the Nucleosome Remodelling and Deacetylation (NuRD) complex. Development 143: 3074-3084.

Palpant NJ, Pabon L, Friedman CE, Roberts M, Hadland B, Zaunbrecher RJ, Bernstein I, Zheng Y, Murry CE. 2017. Generating high-purity cardiac and endothelial derivatives from patterned mesoderm using human pluripotent stem cells. Nat Protoc 12: $15-31$.

Qiu X, Mao Q, Tang Y, Wang L, Chawla R, Pliner H, Trapnell C. 2017. Reversed graph embedding resolves complex single-cell developmental trajectories. Nat Methods 14: 979-982.

Scialdone A, Natarajan KN, Saraiva LR, Proserpio V, Teichmann SA, Stegle O, Marioni JC, Buettner F. 2015. Computational assignment of cell-cycle stage from single-cell transcriptome data. Methods 85: 54-61.

Semrau S, Goldmann JE, Soumillon M, Mikkelsen TS, Jaenisch R, van Oudenaarden A. 2017. Dynamics of lineage commitment revealed by single-cell transcriptomics of differentiating embryonic stem cells. Nat Commun 8: 1096.

Shalek AK, Satija R, Adiconis X, Gertner RS, Gaublomme JT, Raychowdhury R. 2013. Single-cell transcriptomics reveals bimodality in expression and splicing in immune cells. Nature 498: $236-240$.

The Steering Committee of the International Stem Cell Initiative. 2005. The International Stem Cell Initiative: toward benchmarks for human embryonic stem cell research. Nat Biotechnol 23: 795-797.

Streeter I, Harrison PW, Faulconbridge A, The HipSci Consortium, Flicek P, Parkinson H, Clarke L. 2017. The human-induced pluripotent stem cell initiative-data resources for cellular genetics. Nucleic Acids Res 45: D691-D697.

Szklarczyk D, Franceschini A, Wyder S, Forslund K, Heller D, Huerta-Cepas J, Simonovic M, Roth A, Santos A, Tsafou KP, et al. 2015. STRING v10: protein-protein interaction networks, integrated over the tree of life. Nucleic Acids Res 43: D447-D452.

Takahashi K, Yamanaka S. 2006. Induction of pluripotent stem cells from mouse embryonic and adult fibroblast cultures by defined factors. Cell 126: $663-676$

Thomson JA, Itskovitz-Eldor J, Shapiro SS, Waknitz MA, Swiergiel JJ, Marshall VS, Jones JM. 1998. Embryonic stem cell lines derived from human blastocysts. Science 282: 1145-1147.

Tibshirani R. 1996. Regression shrinkage and selection via the lasso. J R Stat Soc Series B Methodol 58: 267-288.

Tibshirani R, Bien J, Friedman J, Hastie T, Simon N, Taylor J, Tibshirani RJ. 2012. Strong rules for discarding predictors in lasso-type problems. J $R$ Stat Soc Series B Stat Methodol 74: 245-266.

Tsankov AM, Akopian V, Pop R, Chetty S, Gifford CA, Daheron L, Tsankova NM, Meissner A. 2015. A qPCR ScoreCard quantifies the differentiation potential of human pluripotent stem cells. Nat Biotechnol 33: 11821192.

van der Maaten L, Hinton GE. 2008. Visualizing data using t-SNE. J Mach Learn Res 9: 2579-2605.

Warde-Farley D, Donaldson SL, Comes O, Zuberi K, Badrawi R, Chao P, Franz M, Grouios C, Kazi F, Lopes CT, et al. 2010. The GeneMANIA prediction server: biological network integration for gene prioritization and predicting gene function. Nucleic Acids Res 38: W214W220.

Weikum ER, Tuntland ML, Murphy MN, Ortlund EA. 2016. A structural investigation into Oct4 regulation by orphan nuclear receptors, germ cell nuclear factor (GCNF), and liver receptor homolog-1 (LRH-1). J Mol Biol 428: $4981-4992$. 
Nguyen et al.

Wen L, Tang F. 2016. Single-cell sequencing in stem cell biology. Genome Biol 17: 71 .

Wilson NK, Kent DG, Buettner F, Shehata M, Macaulay IC, Calero-Nieto FJ, Sanchez Castillo M, Oedekoven CA, Diamanti E, Schulte R, et al. 2015. Combined single-cell functional and gene expression analysis resolves heterogeneity within stem cell populations. Cell Stem Cell 16: 712-724.

Wu G, Feng X, Stein L. 2010. A human functional protein interaction network and its application to cancer data analysis. Genome Biol 11: R53.

Yan L, Yang M, Guo H, Yang L, Wu J, Li R, Liu P, Lian Y, Zheng X, Yan J, et al. 2013. Single-cell RNA-Seq profiling of human preimplantation embryos and embryonic stem cells. Nat Struct Mol Biol 20: 1131-1139.
Yang J, Benyamin B, McEvoy BP, Gordon S, Henders AK, Nyholt DR, Madden PA, Heath AC, Martin NG, Montgomery GW, et al. 2010a Common SNPs explain a large proportion of the heritability for human height. Nat Genet 42: 565-569.

Yang J, Gao C, Chai L, Ma Y. 2010b. A novel SALL4/OCT4 transcriptional feedback network for pluripotency of embryonic stem cells. PLoS One 5: e10766.

Zheng GX, Terry JM, Belgrader P, Ryvkin P, Bent ZW, Wilson R, Ziraldo SB, Wheeler TD, McDermott GP, Zhu J, et al. 2017. Massively parallel digital transcriptional profiling of single cells. Nat Commun 8: 14049.

Received April 11, 2017; accepted in revised form May 3, 2018.

\section{Genome Research}




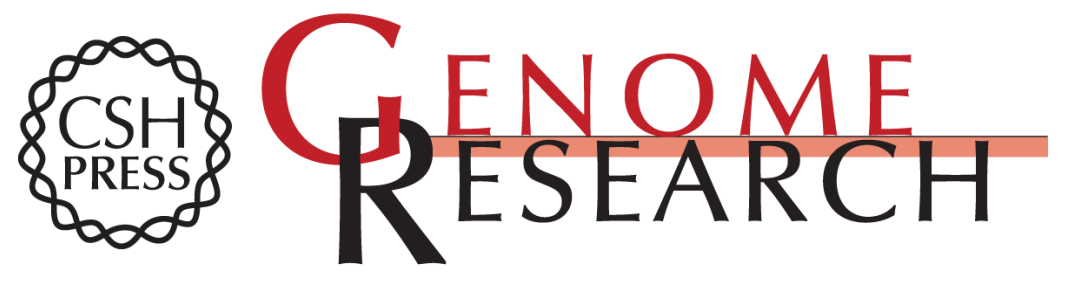

\section{Single-cell RNA-seq of human induced pluripotent stem cells reveals cellular heterogeneity and cell state transitions between subpopulations}

Quan H. Nguyen, Samuel W. Lukowski, Han Sheng Chiu, et al.

Genome Res. 2018 28: 1053-1066 originally published online May 11, 2018

Access the most recent version at doi:10.1101/gr.223925.117

\section{Supplemental http://genome.cshlp.org/content/suppl/2018/06/08/gr.223925.117.DC1 \\ Material}

References This article cites 50 articles, 9 of which can be accessed free at:

http://genome.cshlp.org/content/28/7/1053.full.html\#ref-list-1

Creative This article is distributed exclusively by Cold Spring Harbor Laboratory Press for the Commons

License first six months after the full-issue publication date (see http://genome.cshlp.org/site/misc/terms.xhtml). After six months, it is available under a Creative Commons License (Attribution-NonCommercial 4.0 International), as described at http://creativecommons.org/licenses/by-nc/4.0/. Email Alerting
Service
top right corner of the article or click here.

\section{Affordable, Accurate Sequencing.}

To subscribe to Genome Research go to:

https://genome.cshlp.org/subscriptions 